\title{
Developmental Trajectories of Attention in Normal Korean Population
}

\author{
Han Nah Huh', Sung Hee Kang ${ }^{2}$, Soon Young Hwang ${ }^{3}$, and Hanik K. Yoo ${ }^{1,4}$ \\ ${ }^{1}$ Seoul Brain Research Institute, Seoul, Korea \\ ${ }^{2}$ Happymind Inc., Seoul, Korea \\ ${ }^{3}$ Department of Computer Science and Engineering, Seoul National University, Seoul, Korea \\ ${ }^{4}$ Department of Teacher Education, College of Education, Konkuk University, Seoul, Korea
}

\begin{abstract}
Objectives: This study aimed to investigate the trajectory of change of diverse attention and working memory in Koreans from 4 to 40 years of age.

Methods: The data of 912 subjects from 4 to 15 years of age obtained from a previous standardization study of the computerized comprehensive attention test were merged with the newly obtained data of 150 subjects aged 16 to 40 years from this study. We evaluated the various kinds of attention, in which each subtest had five indicators. Working memory, with parameters such as number of correct responses and span, was also measured.

Results: Our findings indicated that attention developed as age increased, and it decreased or was maintained after a certain age. Selective and sustained attention developed rapidly in children and adolescents, until mid-teens or $20 \mathrm{~s}$ when it ceased development. Divided attention, however, developed up to approximately age 20. In addition, working memory developed until mid-teens or $20 \mathrm{~s}$. Conclusion: We presented the standardized data on diverse kinds of attention and working memory in children, adolescents, and adults in Korea. We could recognize any patterns of change in attention and working memory with increasing age.
\end{abstract}

Key Words: Attention; Continuous performance task; Working memory; Neuropsychologic test; Development.

Received: November 14, 2018 / Revision: December 24, 2018 / Accepted: January 4, 2019

Address for correspondence: Hanik K. Yoo, Seoul Brain Research Institute, 10 Gangbyeonyeok-ro 4-gil, Gwangjin-gu, Seoul 05116, Korea

Tel: +82-2-452-2105, Fax: +82-2-6280-2163, E-mail: hanikyoo@gmail.com

\section{INTRODUCTION}

Attention is one of the fundamental higher cognitive abilities and cannot be explained by a single concept. Although neuroscientists and clinicians have suggested divergent opinions about its definition and scope, several classifications have been widely used to categorize the functions and phenomena of attention. These include: 'focused attention' that indicates the degree of attention by a specific stimulus, 'selective attention' that means paying attention to a stimulus among several stimuli, 'sustained attention' that means continuously maintaining attention to the stimulus until the given task has been completed, and 'divided attention' that gives attention to several stimuli at the same time [1]. Attention is a lengthy process, from preparation for concentration on the given stimuli, to understanding the meaning or value of the stimuli, and to choosing the appropriate stimuli according to the context. Thus, attention is important in completing

This is an Open Access article distributed under the terms of the Creative Commons Attribution Non-Commercial License (https://creativecommons.org/licenses/by-nc/4.0) which permits unrestricted non-commercial use, distribution, and reproduction in any medium, provided the original work is properly cited. information processing [2].

Working memory is a cognitive capability that not only stores, but also processes information for a short period of time. It occurs in a context-oriented manner according to incoming stimuli or previously stored information [3]. Working memory is one of the crucial factors in the performance of executive function [3], which is defined as the ability to plan, organize information, and solve problems appropriately. There have been studies on the interactions between working memory and attention. Attention acts as a 'gatekeeper' for working memory by choosing and encoding information that is relevant to current processing goals. In addition, attentional processes help in the active maintenance of information when spatial working memory is employed [4].

A lack of attention and working memory leads to difficulties in high-level functions such as self-management and learning. Since attentional deficits are a common symptom of many mental disorders [1], evaluation of attention has been used to determine the diagnosis, progress, and sequel of diverse mental disorders [5]. In particular, attention-deficit/hyperactivity disorder (ADHD) is a neurodevelopmental 
disorder characterized by attention deficit, hyperactivity, and impulsivity. The prevalence of ADHD in school-aged children is known to be approximately 5\% [6]. Patients with ADHD suffer from academic and social difficulties and as attention changes naturally through aging, ADHD symptoms are also altered. However, at least $60 \%$ of ADHD symptoms last into adulthood [7]. In order to determine objective levels of attention in people with or without attention deficits, it is necessary to use standardized normative data from children to adults.

Although controversy remains, the most common method to evaluate attention is the computer-based continuous performance test (CPT), which measures correct response count, omission error, commission error, and reaction time to the target stimuli. The test is known to be useful to distinguish children with ADHD from neurotypical children and to monitor drug effects [8]. However, it has some limitations, especially when assessing children with strong intellectual abilities or seniors [9].

The computerized comprehensive attention test (CAT) evaluates various and comprehensive types of attention and working memory in children and adolescents. The CAT was standardized and has been used in Korea [10]. Since it is computerized, the CAT demonstrates higher inter-rater reliability and offers more accurate and sensitive data than pencil and paper tests about response time. The CAT is composed of six subtests to measure focused attention, attentional capacity, sustained attention, divided attention, and visuospatial working memory. These subtests have been widely used to evaluate both normal and ADHD groups [11,12]. In addition, the sustained attention and working memory tests have been known to be effective in screening children with ADHD [13]. Although the CAT has been used to evaluate different levels of attention in Korea [14], it is standardized only for ages 4-15.

Attention typically develops gradually with age, stops in adolescence, remains until a certain age, and decreases along with the decline of brain function. However, we have limited data on the age-related trajectories of various types of attention in Korea, as the normative data of late adolescence and adults have not yet been obtained.

Therefore, in the present study, we administered the CAT to older adolescents and adults and merged the data with previous normative data of children and young adolescents. The complete data were then analyzed to identify how attention and working memory change with age.

\section{METHODS}

\section{Participants}

We used both the previous CAT standardization data from
2009 and new data for this study. The previous data had been collected from 912 elementary, middle, and high school students aged 4 to 15 , excluding those with intellectual disabilities, schizophrenia, other psychoses, organic mental disorders, and vision or hearing problems in Seoul and the metropolitan area [10]. Additional CAT data from 150 healthy subjects aged 16 to 40 and without exclusion conditions were obtained in Seoul and the metropolitan area. Finally, the data of 1062 people [552 males (52.0\%) and 510 females (48.0\%)] were analyzed (Table 1).

\section{Materials}

\section{The computerized CAT}

The CAT was used to evaluate the attention and working memory of study subjects. The CAT consists of selective attention (visual and auditory), sustained attention to response, flanker, divided attention, and spatial working memory tests. The tests have been standardized for children aged 4 to 15 and confirmed to be reliable and valid [9,14]. In the selective attention test, the subject presses the button as quickly as they see/hear the given targets. In the sustained attention test to response task (SA), the subject presses the button when a picture appears, unless it is an 'X.' In the flanker test, the subject responds to stimuli in the center box while ignoring the interference boxes. In the divided attention test (DA), the subject remembers the picture and sound of the previous target and presses the button when the previous target reappears. In the spatial working memory test, the subject remembers the sequence and locations the highlighted boxes, then presses

Table 1. Demographic data of study subjects

\begin{tabular}{cccc}
\hline $\begin{array}{c}\text { Age/age } \\
\text { group (years) }\end{array}$ & $\begin{array}{c}\text { Male } \\
\text { (number) }\end{array}$ & $\begin{array}{c}\text { Female } \\
\text { (number) }\end{array}$ & $\begin{array}{c}\text { Total } \\
\text { (number) }\end{array}$ \\
\hline 4 & 27 & 24 & 51 \\
5 & 31 & 30 & 61 \\
6 & 52 & 41 & 93 \\
7 & 54 & 51 & 105 \\
8 & 67 & 56 & 123 \\
9 & 31 & 40 & 71 \\
10 & 37 & 25 & 62 \\
11 & 29 & 31 & 60 \\
12 & 37 & 29 & 66 \\
13 & 44 & 33 & 77 \\
14 & 48 & 28 & 76 \\
15 & 29 & 38 & 67 \\
$16-19$ & 11 & 15 & 26 \\
$20-29$ & 28 & 34 & 62 \\
$30-39$ & 12 & 14 & 26 \\
$40-49$ & 552 & 210 & 36 \\
Total & & 56 & 62 \\
\hline
\end{tabular}


the boxes in the order they appear and in the reverse order.

Except for the working memory test, each subtest had five indicators [commission errors (CE), omission errors (OE), mean reaction time (RT mean), standard deviation of reaction time (RT sd), and sensitivity coefficient (d')]. The CE indicates the number of responses to the stimuli of the nontarget, and the OE indicates the number of missed responses to the target stimuli. The RT mean refers to the average time taken to respond to the target stimuli, and the RT sd reflects the response time variability to the target stimuli. The d' reflects how effectively the target stimuli are distinguished from the non-target stimuli. The high point of the d' suggests a high level of signal-noise differentiation. The spatial working memory task evaluates the degree of recall of the sequence of the visual stimuli in order and in reverse order and has two indicators (correct response number and memory span).

\section{Procedure}

This study was approved by the Institutional Review Board at Konkuk University (No. 7001355-201711-E-059). The CAT required 40 to 60 minutes to complete.

\section{Analysis}

The data were analyzed using SPSS for Windows (version 21.0; IBM Corp., Armonk, NY, USA). Standardization data were described, and statistical significance was applied at $\mathrm{p}<0.05$. SigmaPlot (version 14.0; Systat Software Inc., San Jose, CA, USA) and Origin ${ }^{\circledR}$ (version 2018b; OriginLab Corp., Northampton, MA, USA) were used to obtain regression equations and graphs of all parameters.

\section{RESULTS}

The normative data of the CAT for 1062 Korean children, adolescents, and adults are presented in Table 2 and 3. The mean and standard deviation (SD) of the OE, CE, RT mean, $\mathrm{RT}$ sd, and d' values by age and gender are also shown.

The graphs of the regression model showed that the relationship between age and task results was non-linear (Supplementary Figs. 1-17 in the online-only Data Supplement). In order to find the changes of attention with age, we described to five indicators of subtests, except in the spatial working memory test. The working memory test was described separately because of different types of indicators.

\section{Visual selective attention test (VSA) and auditory selective attention test (ASA)}

\section{$\mathrm{CE}$ and $\mathrm{OE}$}

The CE and OE of the visual selective attention test (VSA)

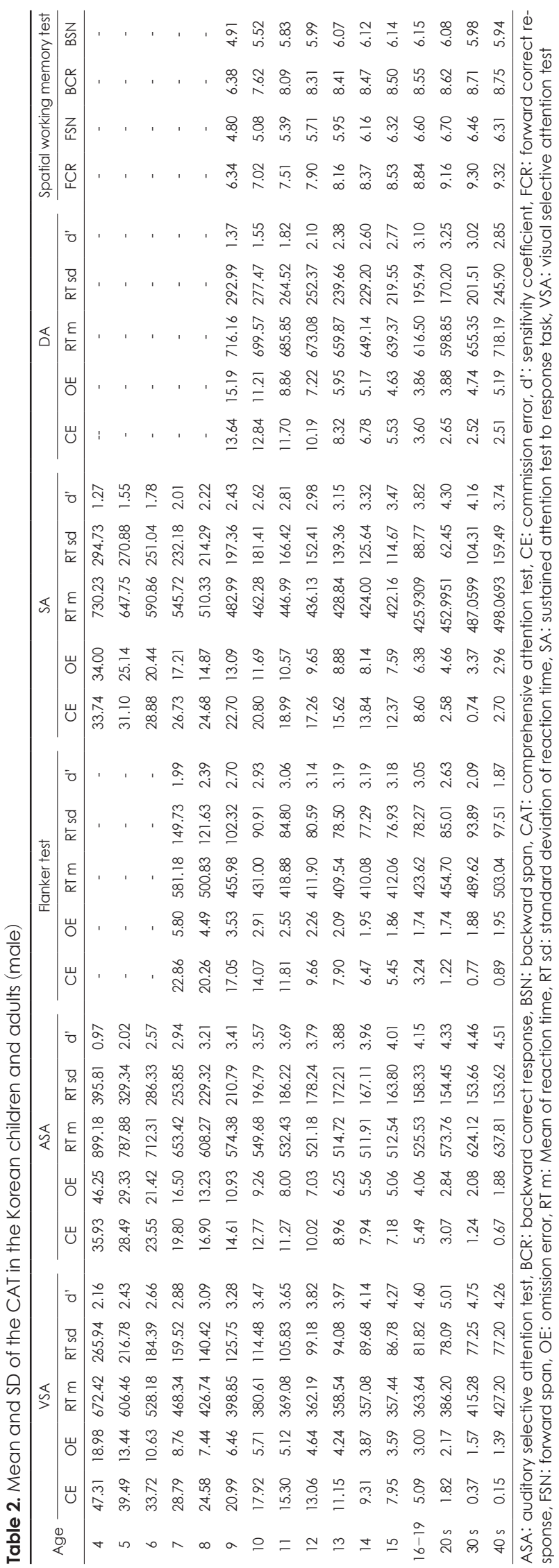




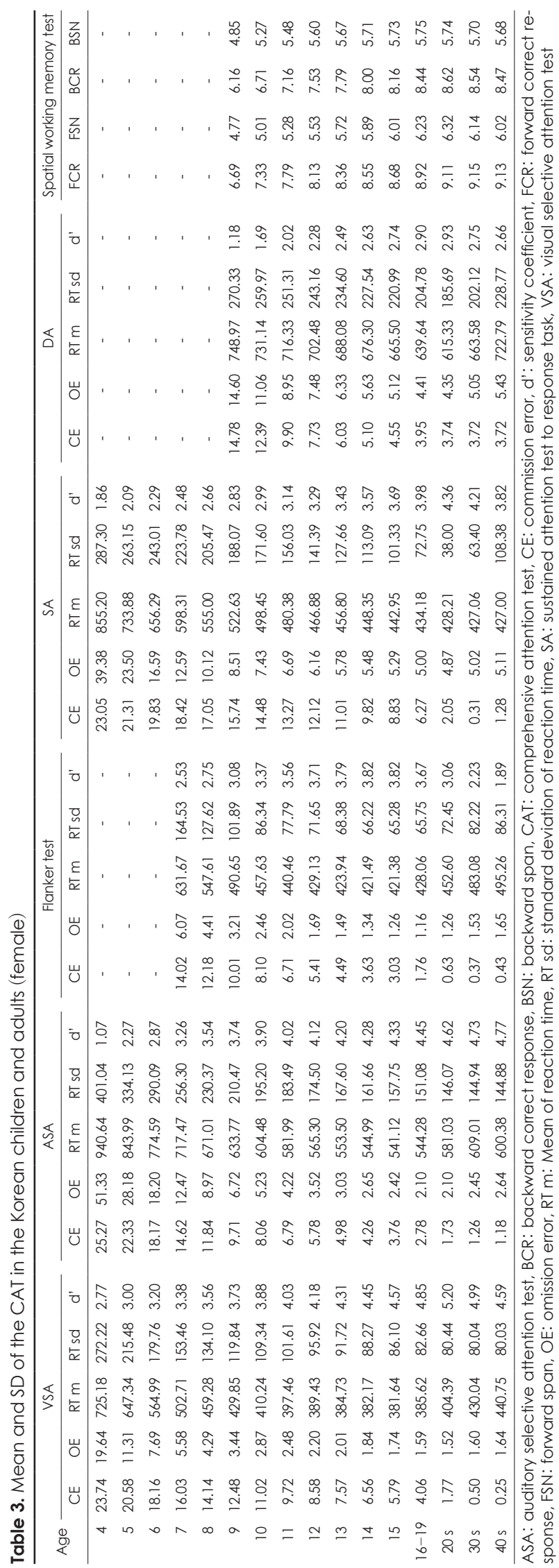

and auditory selective attention test (ASA) had a similar tendency with concave downward shaped graphs. In the VSA, the $\mathrm{CE}$ and $\mathrm{OE}$ were at the maximum points at age 4 in both males and females (CE: $\mathrm{M}=47.31, \mathrm{~F}=23.74$; $\mathrm{OE}: \mathrm{M}=18.98$, $\mathrm{F}=19.64)$. For males, both the $\mathrm{CE}$ and $\mathrm{OE}$ decreased with age, whereas only the $\mathrm{CE}$ decreased for females. Although errors declined sharply until ages $16-19$, they remained stable past those ages. The $\mathrm{OE}$ of females decreased with age and was the lowest in the $20 \mathrm{~s}(\mathrm{OE}=1.52)$ (Supplementary Fig. 1 in the online-only Data Supplement). In the ASA, both the CE and $\mathrm{OE}$ were at their maximum at age 4 (CE: $\mathrm{M}=35.93, \mathrm{~F}=$ 25.27; OE: $\mathrm{M}=46.25, \mathrm{~F}=51.33$ ). They also decreased with age, and declined slightly after ages $16-19$. However, the $\mathrm{OE}$ of females was at its minimum at ages $16-19(\mathrm{OE}=2.10)$, then increased slightly (Supplementary Fig. 4 in the online-only Data Supplement).

\section{RT and RT sd}

The RT and RT sd of the VSA showed a similar tendency to the RT and RT sd of the ASA. In the VSA, the RT mean at age 14 and 15 were the fastest for males and females, respectively ( $\mathrm{M}=357.08 \mathrm{~ms}, \mathrm{~F}=381.64 \mathrm{~ms})$. The RT sd decreased with age; it declined sharply until early teenage years but remained almost unchanged after (Supplementary Fig. 2 in the online-only Data Supplement). In the ASA, the RT at age 14 was the fastest for males $(511.91 \mathrm{~ms})$ and age 15 for females (541.12 ms). The RT sd decreased until age 10 and showed little change after that (Supplementary Fig. 5 in the onlineonly Data Supplement). In the VSA, females responded slower than males ( $t=-3.253, p=0.001)$, while no significant difference was found in the ASA.

\section{d'}

In the VSA, the mean of d' increased until approximately age 20 in both males and females, which was followed by a decrease (Supplementary Fig. 3 in the online-only Data Supplement). In the ASA, though the d' increased with age, the development was rapid until the early teenage years, and then increased slightly (Supplementary Fig. 6 in the online-only Data Supplement).

\section{Flanker test}

\section{$\mathrm{CE}$ and $\mathrm{OE}$}

The flanker test was administered to subjects aged 7 and older. The youngest participants showed the most errors (CE: $\mathrm{M}=22.86, \mathrm{~F}=14.02$; $\mathrm{OE}: \mathrm{M}=5.80, \mathrm{~F}=6.07)$. The $\mathrm{CE}$ decreased sharply until ages $16-19$ and declined slightly until the approximately age $30(\mathrm{M}=0.77, \mathrm{~F}=0.37)$. The males demonstrated significantly higher $\mathrm{CE}$ than the females $(\mathrm{t}=4.981, \mathrm{p}=0.000)$. 
The males and females aged 16 to 19 showed the lowest OE $(\mathrm{M}=1.74, \mathrm{~F}=1.16)$ (Supplementary Fig. 7 in the online-only Data Supplement).

\section{RT and RT sd}

There was a similar tendency between the graphs of the RT and RT sd in the flanker test. The RT mean at age 7 was the slowest $(M=581.18, F=631.67)$, but males at age 13 and females at age 15 were the fastest $(M=409.54, F=421.38)$. Similarly, the SDs in both the males and females were the highest at age $7(M=149.73, \mathrm{~F}=164.53)$ and the lowest at age $15(\mathrm{M}=$ 76.93, F=65.28) (Supplementary Fig. 8 in the online-only Data Supplement).

d'

The minimum points were at age $7(\mathrm{M}=1.99, \mathrm{~F}=2.53)$. The peaks were at age 13 for males and age 14 for females $(M=3.19$, $\mathrm{F}=3.82$ ), then they sharply decreased (Supplementary Fig. 9 in the online-only Data Supplement). The d' of females was significantly higher than that of males $(t=-3.565, \mathrm{p}<0.001)$.

\section{Sustained attention test to response task (SA)}

\section{$\mathrm{CE}$ and $\mathrm{OE}$}

The CE and OE of the SA showed the maximum point was at age 4 (CE: $\mathrm{M}=33.74, \mathrm{~F}=23.05$; $\mathrm{OE}: \mathrm{M}=34.00, \mathrm{~F}=39.38$ ). The CE sharply dropped until age 16 to 19 , where it decreased slightly, and then increased again. The OE in males decreased with age; however, in females, it was its minimum in their 20 s $(\mathrm{OE}=4.87)$ and remained stable (Supplementary Fig. 10 in the online-only Data Supplement).

\section{RT and RT sd}

In both males and females, the RT mean was the slowest at age $4(\mathrm{M}=730.23, \mathrm{~F}=855.20)$. The $\mathrm{RT}$ of males was fastest at age $15(\mathrm{RT}=422.16)$, and in females, there was a sharp decline until mid-teenage years, which then decreased slightly. The RT sd was lowest in the 20s for both males and females $(\mathrm{M}=62.45, \mathrm{~F}=38.00)$ (Supplementary Fig. 11 in the onlineonly Data Supplement).

\section{d'}

The mean of the d' increased until the 20s $(\mathrm{M}=4.30, \mathrm{~F}=4.36)$, which was followed by a decrease (Supplementary Fig. 12 in the online-only Data Supplement).

\section{Divided attention test (DA)}

\section{$\mathrm{CE}$ and $\mathrm{OE}$}

The DA was applied to subjects aged 9 years and older. Both the $\mathrm{CE}$ and $\mathrm{OE}$ had a peak at age 9 (CE: $\mathrm{M}=13.64, \mathrm{~F}=14.78$; $\mathrm{OE}: \mathrm{M}=15.19, \mathrm{~F}=14.60)$. The $\mathrm{CE}$ decreased with age but stayed at a similar level after approximately age 20 , whereas the OE had a minimum point at ages 16-19 for males and in the 20s for females $(M=3.86, F=4.35)$ (Supplementary Fig. 13 in the online-only Data Supplement).

\section{RT and RT sd}

At age 9, the RT mean was the slowest $(\mathrm{M}=716.16, \mathrm{~F}=$ 748.97), while the fastest was at approximately age $20(\mathrm{M}=$ $598.85, \mathrm{~F}=615.33)$. The RT gradually increased with age following their 20s. The tendency of the RT sd was similar to that of the RT, which decreased until their 20s $(M=170.20$, $\mathrm{F}=185.69$ ) (Supplementary Fig. 14 in the online-only Data Supplement).

\section{d'}

The mean of the d' increased until approximately age 20 in both males and females $(\mathrm{M}=3.25, \mathrm{~F}=2.93)$, and decreased gradually with age after that (Supplementary Fig. 15 in the online-only Data Supplement).

\section{Spatial working memory test}

\section{Forward}

The graphs of the correct response number and memory span were shaped concave downward. In males, though the correct response gradually increased with age, it remained stagnant from the 20s. In females, it was the highest in the 30s (Supplementary Fig. 16 in the online-only Data Supplement). The memory span was the widest in the $20 \mathrm{~s}(\mathrm{M}=6.70$, $\mathrm{F}=6.32$ ). The graphs then flattened out (Supplementary Fig. 17 in the online-only Data Supplement).

\section{Backward}

The correct response and memory span of the backward test were similar to the forward test. In males, the correct response drastically increased until mid-teenage years and increased slightly after that, becoming stable. The highest correct response in females was in their 20s. The largest span was at ages 16-19 for males and females $(M=6.15, F=5.75)$. After the peaks, the values remained steady (Supplementary Figs. 16 and 17 in the online-only Data Supplement).

\section{DISCUSSION}

Through this study, we acquired the standardized data on various kinds of attention and working memory in children, adolescents, and adults in Korea. Furthermore, we observed how attention and working memory change with age. 


\section{VSA and ASA}

Although the VSA and ASA, as well as the flanker test, revealed selective attention capability [10], there might be some differences according to the results of the present study. Both the CE and OE in the VSA declined with age and showed a ceiling effect after approximately age 20 (Supplementary Fig. 1 in the online-only Data Supplement); however, the OE might be lower than the CE. Additionally, female teenagers had a tendency of lower $\mathrm{CE}$ than males. In the ASA, we also observed a similar tendency (Supplementary Fig. 4 in the online-only Data Supplement). Many studies using the CPT, on which the VSA and ASA were based, have reported analogous results to this study. In a study of 816 children and adolescents between the ages of 9 and 17, both the $\mathrm{OE}$ and $\mathrm{CE}$ tended to decrease with age [11]. The same tendency was also observed in the study of 514 girls aged 5 to 12 [15]. A cohort study that tracked the CPT performance of children at age $8(n=435)$ also showed that the OE decreased rapidly after age 6 . In particular, the most rapid changes were shown between ages 8 and 10, with more gradual changes occurring between ages 10 and 13 [16]. This pattern was similar to results from our study. A CPT study in adults showed relatively high levels of the correct responses, which corresponded with our study [17].

In the VSA, the RT decreased until early teenaged years and then increased with age. In only the VSA, females responded slower than males. The RT sd of the VSA and ASA were drastically reduced until ages 14 and 15 , then maintained at a similar level. In the previous CPT studies of children and adolescents, the RT and RT sd decreased with age $[11,15]$, and females showed longer RT than males, which is similar to our study (Supplementary Figs. 2 and 5 in the online-only Data Supplement) [11].

The d' of the VSA reached the highest value in the mid-20 $s$ and gradually declined after that, while the curve of the d' in the ASA sharply increased up to age 12 (Supplementary Figs. 3 and 6 in the online-only Data Supplement). In a previous CPT study of children and adolescents, the d'value increased with age, which is consistent with our study $[11,15,18]$. When comparing adolescents (mean age $=14.0$ years) to adults (mean age $=41.3$ years), adolescents had higher $d^{\prime}(p<$ 0.01 ) [17]. Normally the $d$ ' is a good parameter of selective attention because it implies how the subject distinguishes the target from the non-target stimuli [10]. Thus, these findings indicate that selective attention develops rapidly in children and adolescents and minimally develops or even decreases from the late teenaged years to early 20 s. In the ASA, however, the d' sharply increased until age 12 , and gradually increased after that. This result was contrary to a precedent study, which used a visual task, reporting higher d' in ado- lescents [17]. This distinction might be a result of the different tasks used; however, because there has not been a study that uses auditory selective stimuli, further studies are needed to obtain confirmative results. There was also a study showing that females have significantly higher values than males in the d' [11]. In this study, females had higher values than males; however, this was not a statistically significant difference.

Females are thought to perform better and more cautiously than males because the female's d' and RT mean were higher in the selective attention tests. This could be related with the well-known fact that boys with ADHD have more hyperactive and impulsive symptoms and comorbid externalizing disorders than girls [19]. However, further research is necessary to obtain more certain results.

\section{Flanker test}

The flanker test is an effective test for measuring interference-selective attention, and it has been known to effectively distinguish ADHD from normal children [20]. The CE in males dropped sharply by ages $16-19$, and gradually decreased after that. The CE of males was higher than that of females, decreased slightly until mid-20s, and remained stable after approximately age 30 . The $\mathrm{OE}$ in both males and females decreased rapidly until age 10, staying almost unchanged after that (Supplementary Fig. 7 in the online-only Data Supplement). The tendency of the RT was similar to that of the VSA. It decreased until ages 13 and 14, then gradually increased in both males and females. The RT sd declined sharply and gradually increased after teenaged years (Supplementary Fig. 8 in the online-only Data Supplement). For d', females were higher than males, and the peaks of d' were reached at ages 16-19 in males and at age 14 in females, then declined in both (Supplementary Fig. 9 in the online-only Data Supplement). Higher CE and lower d' in males than females could be due to the males' more impulsive tendencies. A previous flanker test study of the ages $20-70$ revealed that the RT increased with age, but the error rate did not change significantly [21]. It implied that though old age could be related to slow speed, it would not be associated with accuracy. Similarly, the CE of the ages 20-40 in our study was rarely variable.

\section{SA}

The CPT, such as the SA, VSA, and ASA, that measured sustained attention has been widely used to evaluate patients with ADHD [11,12]. Though the application should be conducted with caution, sustained attention could be effective in screening children with ADHD [18].

In the present study, the CE of the SA gradually decreased until approximately age 30 , and slightly increased after that. 
However, the OE decreased relatively quickly until age 10, and then was maintained (Supplementary Fig. 10 in the onlineonly Data Supplement). As CE is known to be related to a lack of inhibition against impulsivity [22], inhibition likely develops into the 30s. Carriere et al. [23] also suggested that sustained attention developed into early adulthood and the OE decreased with age but rarely changed after the 20s. Other studies on impulse control in adults suggested that though age and impulse control were related, the inverse correlation between them were not [24]. These arguments could be supported by our finding that the CE decreased until the 30 s and increased after that, but the $\mathrm{OE}$ was steady from teenaged years.

The RT mean sharply decreased until the mid-teenaged years, and then increased slowly in males and females. The RT sd resembled a U-like shape in which the nadir was the mid-20s (Supplementary Fig. 11 in the online-only Data Supplement). The d' was the highest in the late 20s and gradually declined after that (Supplementary Fig. 12 in the online-only Data Supplement). The d' of the other tests in the CAT reached the peaks in the teenaged years to the 20s, which were earlier than that of the SA. According to previous studies, the reaction speed, errors, and accuracy of the sustained attention increased with age [25] and the error-processing brain activation increased continuously into adulthood [26].

\section{DA}

The CE of the DA decreased until the mid- to late-teenaged years, and stayed stable after that; however, the OE decreased until the mid-teenaged years and steadily increased from the late-teenaged years and the 20s in males and females (Supplementary Fig. 13 in the online-only Data Supplement). The RT changed to a U-like shape, which decreased until the mid-20s and increased after that. The RT sd also showed a similar tendency, decreasing in the 20s, and then increased rapidly (Supplementary Fig. 14 in the online-only Data Supplement). The d' reached its peak in the 20 s and gradually declined after that (Supplementary Fig. 15 in the online-only Data Supplement).

There have been few studies on the divided attention of children and adolescents. In a study of adults, elderly adults (ages 61-80) had a significantly lower level of divided attention capacity in comparison with younger (ages 21-37) and middle-aged (ages 40-58) adults, and the young and middleaged adults did not differ in the divided attention [27]. A recent study on multitasking found that younger adults performed better than older adults, but the difference in the results was not caused only by age (e.g., development environment familiar with digital machines) [28]. The results of these previous studies were in accordance with ours.

\section{Spatial working memory test}

The graphs of the correct responses in the forward and backward tasks were similar. The correct responses of both males and females increased sharply until the 20s and showed a ceiling effect after that (Supplementary Fig. 16 in the online-only Data Supplement). Both the forward and backward spans quickly climbed in the late teenaged years and 20s, and decreased after that (Supplementary Fig. 17 in the online-only Data Supplement). There have been many studies where working memory has been found to gradually develop throughout childhood and into adolescence. A study on the development of the spatial working memory for the ages 4-15 also reported that linear functions increased with age [29]. However, a study of working memory span for ages 20-70 revealed an age-related reduction in working memory span [30]. The results of the present study were consistent with these results, and it appeared that working memory developed from the mid-teenaged years to the 20 s, and then gradually slowed.

There were several limitations to this study. First, there was a lack of representation because the standardization data were collected for children, adolescents, and adults in only Seoul and Gyeong-gi Province. To compensate for that, additional supplementation of people residing in other regions is necessary. Second, the clinical usefulness of the CAT in an adult population has not been confirmed because the clinical study on adult ADHD has not yet been conducted in Korea. Further studies will need to confirm whether the CAT would be useful for screening and diagnosing adults with ADHD.

Nevertheless, this is the first study on how various kinds of attention and working memory change with age from children to adults in Korea. The standardized data from this study could be used to obtain objective attention and working memory data through normal and clinical groups with mental health conditions, including ADHD, in Korea.

\section{CONCLUSION}

The study provides the normative data and developmental trajectories on varying types of attention and working memory for children, adolescents, and adults in Korea, from the ages 4-40 through a computerized test tool. The findings will be available in the future for clinical and research material for various clinical groups, including $\mathrm{ADHD}$, as well as for normal populations. 


\section{Supplementary Materials}

The online-only Data Supplement is available with this article at https://doi.org/10.5765/jkacap.180033.

\section{Conflicts of Interest}

The authors have no potential conflicts of interest to disclose.

\section{REFERENCES}

1) Cohen RA. Attentional dysfunction associated with psychiatric illness. In: Cohen RA, editor. The Neuropsychology of Attention 2nd. New York: Plenum Press 1993. p.275-305.

2) Ha KS, Kang UG, Kim JH. Attention and psychiatric disorders. Korean J Biol Psychiatry 1997;4:19-23.

3) Baddeley A. Working memory. Science 1992;255:556-559.

4) Awh E, Vogel EK, Oh SH. Interactions between attention and working memory. Neuroscience 2006;28:201-208.

5) Hong KS, Kim JG, Koh HJ, Koo MS, Kim JH, Lee D, et al. Effects of risperidone on information processing and attention in first-episode schizophrenia. Schizophr Res 2002;53:7-16.

6) Shaffer D, Fisher P, Dulcan MK, Davies M, Piacentini J, SchwabStone ME, et al. The NIMH Diagnostic Interview Schedule for Children Version 2.3 (DISC-2.3): description, acceptability, prevalence rates, and performance in the MECA Study. Methods for the Epidemiology of Child and Adolescent Mental Disorders Study. J Am Acad Child Adolesc Psychiatry 1996;35:865-877.

7) Biederman J, Faraone SV, Mick E, Spencer T, Wilens T, Kiely K, et al. "High risk for attention deficit hyperactivity disorder among children of parents with childhood onset of the disorder: a pilot study." Am J Psychiatry 1995;152:431-435.

8) Corkum PV, Siegel LS. Is the continuous performance task a valuable research tool for use with children with attention-deficit-hyperactivity disorder? J Child Psychol Psychiatry 1993;34:1217-1239.

9) Douglas VI. Attention and cognitive problems. In: Rutter M, editor. Developmental neuropsychiatry. New York: Guilford Press 1983. p. 280-329.

10) Yoo HI, Lee JS, Kang SH, Park EH, Jung JS, Kim BN, et al. Standardization of the comprehensive attention test for the Korean children and adolescents. J Korean Acad Child Adolesc Psychiatry 2009;20:68-75.

11) Conners CK, Epstein JN, Angold A, Klaric J. Continuous performance test performance in a normative epidemiological sample. J Abnorm Child Psychol 2003;31:555-562.

12) Losier BJ, McGrath PJ, Klein RM. Error patterns on the continuous performance test in non-medicated and medicated samples of children with and without ADHD: a meta-analytic review. J Child Psychol Psychiatry 1996;37:971-987.

13) Nigg JT. Neuropsychologic theory and findings in attention-deficit/ hyperactivity disorder: the state of the field and salient challenges for the coming decade. Biol Psychiatry 2005;57:1424-1435.

14) Seo JM, Lee JS, Kim SY, Kim HW. Diagnostic significance of comprehensive attention test in children and adolescents with atten- tion-deficit hyperactivity disorder. J Korean Acad Child Adolesc Psychiatry 2011;22:246-252.

15) Kanaka N, Matsuda T, Tomimoto $Y$, Noda $Y$, Matsushima E, Matsuura M, et al. Measurement of development of cognitive and attention functions in children using continuous performance test. Psychiatry Clin Neurosci 2008;62:135-141.

16) Rebok GW, Smith CB, Pascualvaca DM, Mirsky AF, Anthony BJ, Kellam SG. Developmental changes in attentional performance in urban children from eight to thirteen years. Child Neuropsychol 1997; 3:28-46.

17) Chen WJ, Hsiao CK, Hsiao LL, Hwu HG. Performance of the continuous performance test among community samples. Schizophr Bull 1998;24:163-174.

18) Barkley RA. Behavioral inhibition, sustained attention, and executive functions: constructing a unifying theory of ADHD. Psychol Bull 1997;121:65-94.

19) Hasson R, Fine JG. Gender differences among children with ADHD on continuous performance tests: a meta-analytic review. J Atten Disord 2012;16:190-198.

20) Seo JM, Kim HY, Yeo JY, Byun EH, Chung SH. Executive function in attention-deficit/hyperactivity disorder: relationship of comprehensive attention, stoop color-word, children's color trails, and Wisconsin card sorting tests. J Korean Neuropsychiatr Assoc 2012;51: 59-69.

21) Salthouse TA. Is flanker-based inhibition related to age? Identifying specific influences of individual differences on neurocognitive variables. Brain Cogn 2010;73:51-61.

22) Brewis A, Schmidt KL, Casas CAS. Cross-cultural study of the childhood developmental trajectory of attention and impulse control. Int J Behav Dev 2003;27:174-181.

23) Carriere JS, Cheyne JA, Solman GJ, Smilek D. Age trends for failures of sustained attention. Psychol Aging 2010;25:569-574.

24) De Bruin A, Sala SD. Effects of age on inhibitory control are affected by task-specific features. Q J Exp Psychol (Hove) 2018;71:12191233.

25) Betts J, McKay J, Maruff P, Anderson V. The development of sustained attention in children: the effect of age and task load. Child Neuropsychol 2006;12:205-221.

26) Luna B, Padmanabhan A, O'Hearn K. What has fMRI told us about the development of cognitive control through adolescence? Brain Cogn 2010;72:101-113.

27) Ponds RW, Brouwer WH, Van Wolffelaar PC. Age differences in divided attention in a simulated driving task. J Gerontol 1988;43: P151-P156.

28) Bergman O, Tene-Rubinstein M, Shalom J. The use of attention resources in navigation versus search. Pers Ubiquit Comput 2013;17: 583-590.

29) Gathercole SE, Pickering SJ, Ambridge B, Wearing H. The structure of working memory from 4 to 15 years of age. Dev Psychol 2004; 40:177-190.

30) Timothy AS, Babcock RL. Decomposing adult age differences in working memory. Dev Psychol 1997;27:763-776. 